Eos, Transactions, American Geophysical Union, Vol. 75, No. 16, April 19, 1994, Pages 185 and 193.

\section{Anomalous Cosmic Rays: Interstellar Interlopers in the Heliosphere and Magnetosphere}

\author{
R. A. Mewaldt, A. C. Cummings, and E. C. Stone
}

Since the beginning of the space age, it was known that two main sources of energetic particles pervade interplanetary space: galactic cosmic rays (GCRs), which originate in sources outside the solar system, and solar energetic particles (SEPs), associated with transient events on the Sun.

But over 20 years ago, instruments on the Pioneer 10, IMP 5, and IMP 7 spacecraft discovered a third component of energetic particles known as "anomalous cosmic rays," that represents a sample of nearby interstellar material.

Over the past 2 decades, ACRs have been used to study the acceleration and transport of energetic particles within the solar system, deduce the global properties of the he liosphere-the bubble of solar wind that envelopes the solar system, and study the interstellar material itself.

It has recently been shown that some of these ACRs have become trapped in Earth's magnetic field, where they form a radiation belt composed of interstellar material (Figure 1). Also, ACRs are being used to address a question that has existed ever since the discovery of the solar wind: "How large is the heliosphere?"

\section{Discovery of ACRs}

The unusual composition of ACRs led to their discovery in 1973. Pioneer and IMP observations revealed anomalous excesses of several elements in low-energy cosmic rays, including $\mathrm{He}, \mathrm{N}, \mathrm{O}$, and $\mathrm{Ne}$. For example, $\mathrm{O}$ exceeded $\mathrm{C}$ in abundance by about 30 to 1 , and $\mathrm{He}$ was more abundant than $\mathrm{H}$ (see Fig. ure 2). By contrast, in SEPs and GCRs, C and $\mathrm{O}$ are almost equally abundant, and $\mathrm{H}$ is typically $\geq 10$ times more abundant than $\mathrm{He}$.

In 1977, the Pioneer 10 and 11 spacecraft were joined in their journey to the outer solar system by Voyager 1 and 2 . As the four spacecraft moved outward, the number of particles hitting $1 \mathrm{~cm}^{2} / \mathrm{s}$ increased, indicating that ACRs were not accelerated on the Sun.

In addition, the ACR intensity was found to be inversely correlated with the 11-year sunspot cycle, similar to the well-known "so-

220-47 Downs Laboratory of Physics, California Institute of Technology, Pasadena, CA 91125

Copyright 1994 by the American Geophysical Union 0096/3941/7516/94/185/\$01.00. lar modulation" of GCRs. However, while the low-energy GCR intensity varies by a factor of less than ten from solar minimum to solar maximum, depending on particle energy, the intensity of ACRs varies by a factor of $>100$ ! Both observations indicated that the source of ACRs must be well beyond the Pioneer and Voyager spacecraft.

\section{The Origin of ACRs}

Soon after the discovery of ACRs, Fisk et al. [1974] proposed that they represent a sample of particles from interstellar space. To understand this suggestion, the interaction of the interstellar medium (ISM), the vast region between the stars, and the heliosphere must be considered.

As the Pioneer and Voyager spacecraft

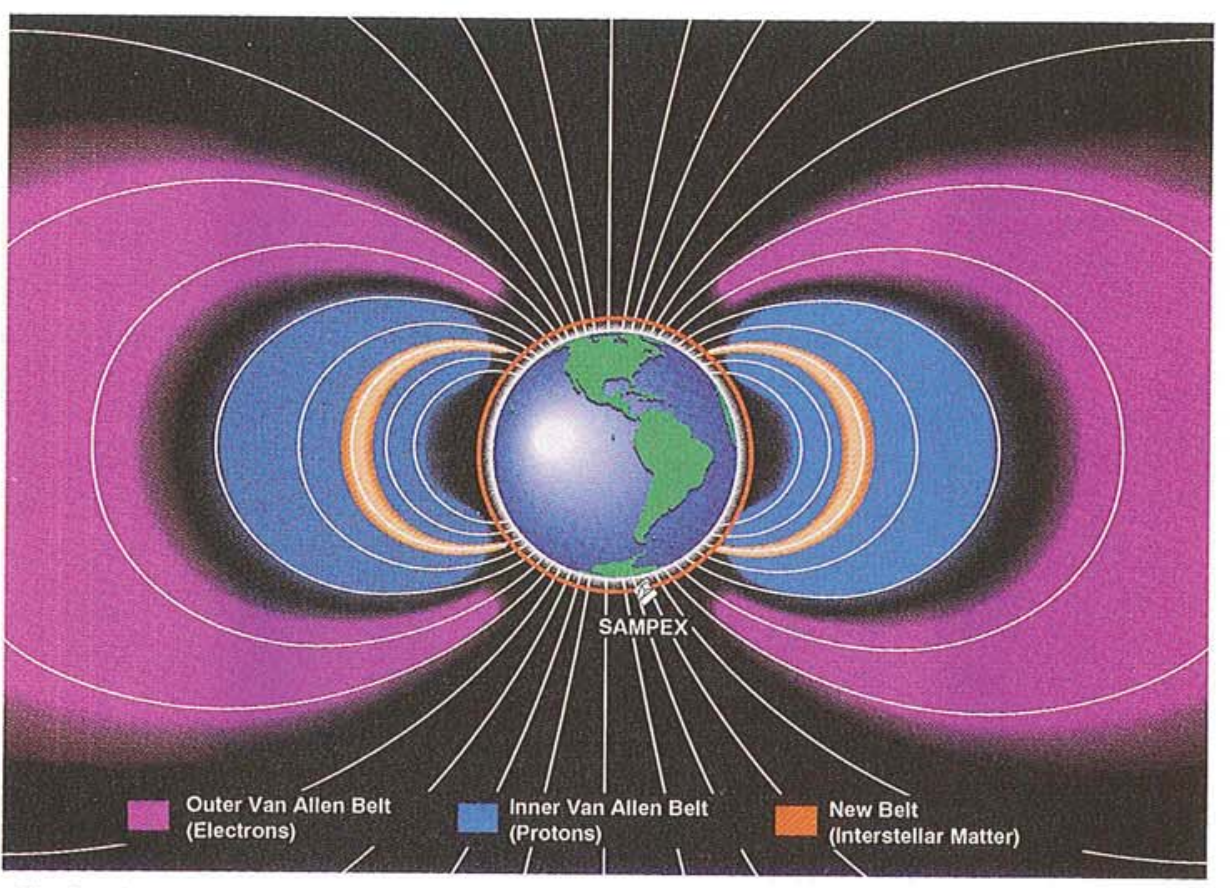

Fig. 1. Schematic cross section of the trapped radiation belts surrounding the Earth. The Van Allen belts are shown in blue and purple. The inner belt is composed mainly of energetic protons, while the outer belt is mainly energetic electrons. A newly identified radiation belt, shown as two bright yellow crescents, is composed of energetic heavy nuclei that originated in the local interstellar medium. All of these belts approach closest to Earth in the south Atlantic region because of the offset of the Earth's magnetic dipole. The orbit of the polar-orbiting SAMPEX satellite, which has been studying the new belt, is indicated. moved to the outer solar system, they studied the solar wind at increasing distances from solar wind is the extension of the Sun's hot corona. It expands at helocity into interplanetary space and is composed of protons, electrons, and heavie speeds of $\sim 400 \mathrm{~km} / \mathrm{s}$.

Embedded in the solar wind is the distended solar magnetic field. As the solar wind expands, it blows a bubble, pushing against the interstellar magnetic field and the hin gas of the local ISM.

Somewhere beyond the orbit of Pluto is an interface called the heliopause, which separates the bubble of solar wind plasma from the ISM (Figure 3). As the solar wind approaches the heliopause, it slows abruptly termination shock.

As the Sun moves through interstellar space, the heliosphere encounters the gas that makes up the ISM. Interstellar ions, having lost one or more of their electrons, are prevented from flowing across the helioHowever, the electrically neutral atoms are unaffected by the magnetic field and can drift into the inner heliosphere, where some of the atoms will be ionized by solar UV radiation or by charge exchange with the solar wind (Figure 4). 


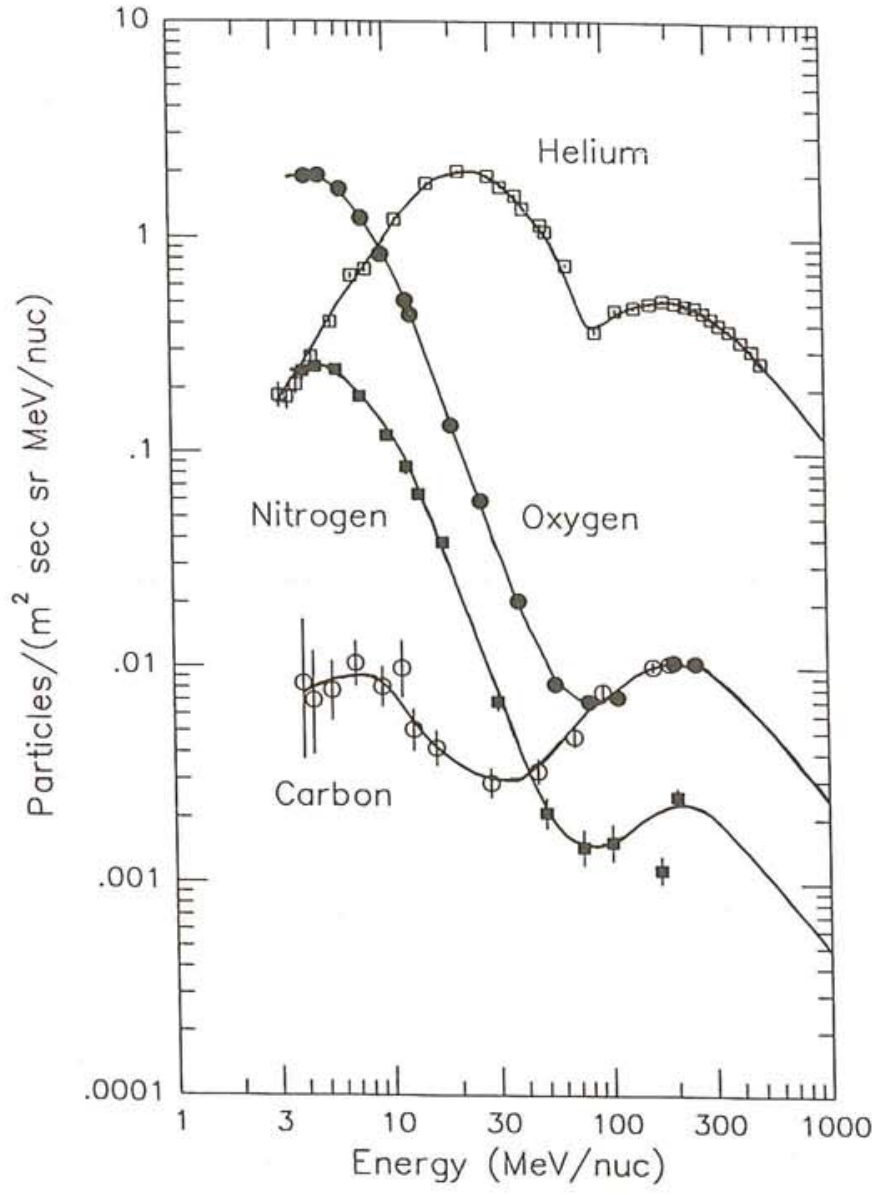

\section{Origin of Anomalous Cosmic Rays}

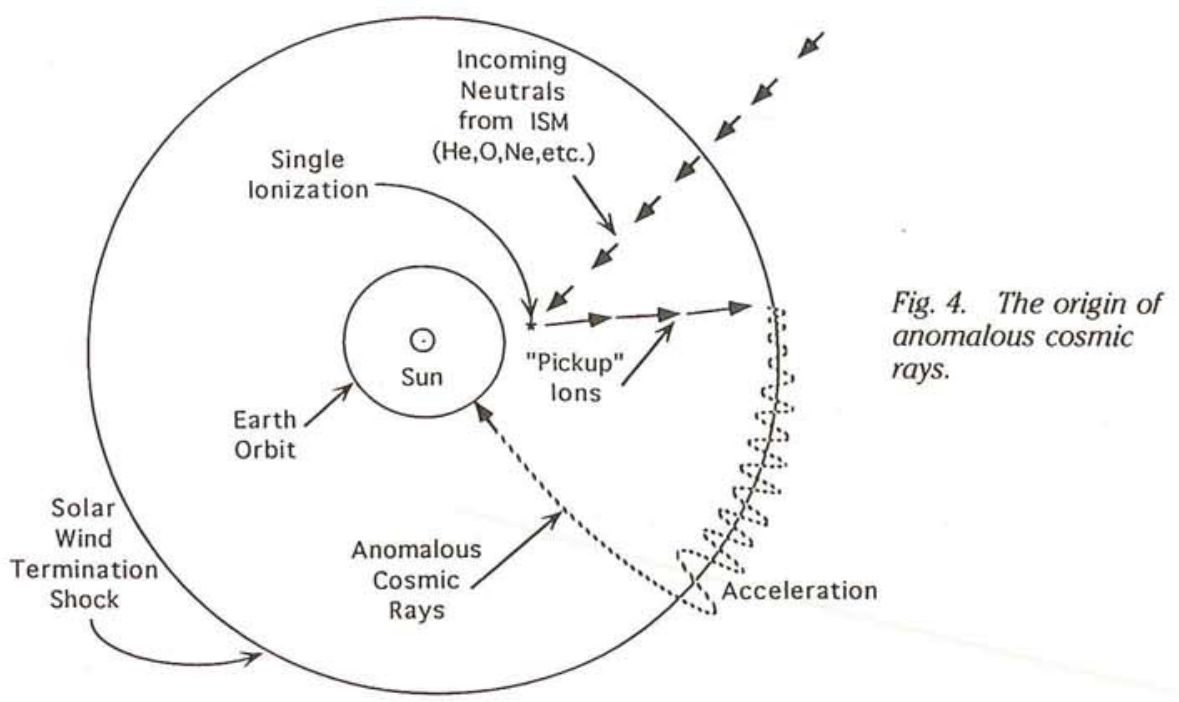

These new ions are then picked up by the solar wind and convected into the outer heliosphere. Fisk et al. [1974] suggested that these "pickup ions" are accelerated to velocities $10-20 \%$ of the velocity of light in the outer heliosphere to become ACRs.

Pesses et al. [1981] proposed that this acceleration takes place at the solar wind termination shock. Once accelerated, some
Fig. 2. Intensity of cosmic ray $\mathrm{He}, \mathrm{C}, \mathrm{N}$, and $O$ nuclei as a function of their measured kinetic energy. These data were measured by instruments on Voyager 2 at a distance of $\sim 23 \mathrm{AU}$ from the Sun during the 1987 solar minimum. At kinetic energies $>100 \mathrm{MeV} / \mathrm{nuc}$, the particles are of galactic cosmic ray origin.

The enhancements in the spectra of $\mathrm{He}, \mathrm{N}$, and $O$ below $\sim 50$ MeVInuc are due to anomalous cosmic rays. A somewhat smaller ACR contribution is also observed for $C$.

Fig. 4. The origin of anomalous cosmic rays.

of these particles diffuse and drift into the inner heliosphere as cosmic rays subject to the same solar cycle modulation as GCRs.

The model described explains the unusual composition of ACRs, because it selects only those elements that are predominantly neutral in the ISM. Atoms that are difficult to ionize, with first ionization potential (FIP) $\geq 13.6 \mathrm{eV}$ (for example, $\mathrm{He}, \mathrm{N}, \mathrm{O}$,
$\mathrm{Ne}$, and $\mathrm{Ar}$ ) are primarily neutral in the ISM, while those with FIP $<13.6 \mathrm{eV}$ (for example, $\mathrm{C}, \mathrm{Mg}, \mathrm{Si}$, and $\mathrm{Fe}$ ) are expected to be ionized to an overwhelming extent.

All of the common high-FIP elements, including $\mathrm{Ar}$ and possibly $\mathrm{H}$, have now been found in ACRs [e.g., Cummings and Stone, 1987]. ACR carbon has also been detected by, but only in very small amounts, as expected if only $\sim 1 \%$ of interstellar $\mathrm{C}$ is in the neutral state. These measurements have been used to obtain the relative abundances of neutral atoms in the local ISM.

The model in Figure 4 predicts that ACRs should be singly charged, having lost only a single electron. By contrast, GCRs have been essentially stripped of electrons during their $\sim 10$ million year passage through the galaxy.

Several aspects of this picture have been verified. The existence of neutral $\mathrm{H}$ and $\mathrm{He}$ flowing into the heliosphere was established in the early 1970s. Recently, the Ulysses spacecraft measured directly neutral $\mathrm{He}$ atoms streaming into the inner heliosphere. In addition, the Active Magnetospheric Particle Tracer Explorer and Ulysses missions have both observed "pick-up" ions with the expected properties [e.g., Gloeckler et al., 1993].

\section{Exploring the Heliosphere with Anomalous Cosmic Rays}

The fleet of Pioneer and Voyager spacecraft provided our first exploratory view of the three-dimensional structure of the heliosphere. One of their key objectives is to locate the termination shock and heliopause, thereby defining the size of the heliosphere.

Recently, an estimate of the distance to the termination shock has been obtained by extrapolating the ACR intensity gradients measured between Voyager 2 at $\sim 20 \mathrm{AU}$ and Pioneer 10 at $\sim 40 \mathrm{AU}$ into the outer heliosphere [Cummings et al., 1993a]. It was found that the time dependence of the ACR fluxes of $\mathrm{He}$ and $\mathrm{O}$ during the 1987 solar minimum period could best be explained if the termination shock was at $67 \pm 5 \mathrm{AU}$.

It is interesting to compare this result with estimates of the distance to the heliopause, based on data from the Voyager plasma wave experiments [Gurnett et al., 1993]. Analysis of radio emissions observed by these experiments, coming from the direction of the nose of the heliopause, suggests a heliopause distance of 116-177 AU in 1992 near solar maximum.

Although these shock and heliopause estimates are for different phases of the solar cycle, they appear to be consistent with models of the heliosphere if the heliopause distance is toward the lower end of this range. These findings suggest that both Pioneer 10 and Voyager 1 may pass through the termination shock by 1997 , when both will be beyond $67 \mathrm{AU}$. If so, these spacecraft will be able to observe, in situ, the acceleration of particles to cosmic ray energies. 


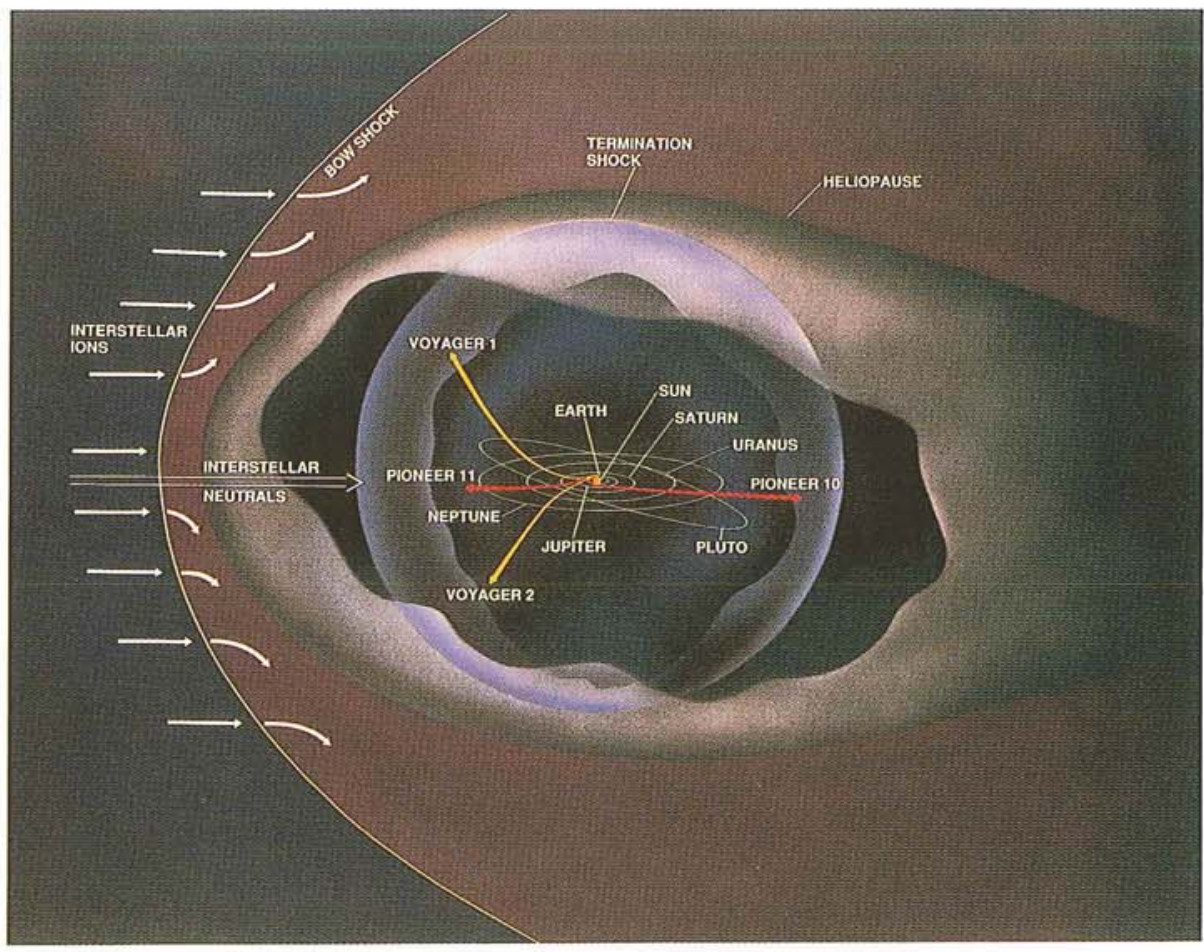

Fig. 3. Schematic illustration of the large-scale structure of the heliosphere. Surrounding the solar wind termination shock is the heliopause, which forms the interface between solar and interstellar plasma, and a possible "bow shock" that may be located beyond the heliopause. The present positions of the Pioneer and Voyager spacecraft are indicated. The termination shock is assumed to be $67 \mathrm{AU}$ from the Sun, as derived from an analysis of anomalous cos mic ray data from Pioneer 10 and Voyager 2.

\section{Trapped ACRs}

The well-known Van Allen radiation belts are regions of intense fluxes of energetic protons and electrons trapped in Earth's magnetic field. Blake and Friesen [1977] predicted that if ACRs are indeed singly charged, they could also become trapped in the magnetosphere, forming a radiation belt of trapped ACRs.

Because a singly charged oxygen ACR has a radius of gyration eight times larger as it spirals in the Earth's magnetic field, it can penetrate to lower latitudes than if it were fully stripped. If it were then to skim the atmosphere, the ACR oxygen ion would be stripped of all its electrons, reducing its radius of gyration and leaving it to spiral back and forth from one hemisphere to another.

The first evidence for the existence of trapped ACRs was provided by a team of Russian and U.S. scientists using observations from a series of COSMOS satellites launched from 1985 to 1988 [Grigorov et al., 1991]. The detectors on COSMOS could not directly measure the location of the trapped $O$ because there was no record of where or when the particles were observed during the spacecraft orbit. However, they did reveal that the intensity of trapped $\mathrm{O}$ tracked the interplanetary intensity of ACR nuclei measured by IMP 8, increasing from 1986 to early 1987, and then decreasing dramatically in intensity with the onset of solar activity in mid-1987. prediction and the COSMOS observations, the SAMPEX data show that the location of the belt is much closer to the Earth than expected and that it is much narrower.

In essence, the new radiation belt corresponds to a magnetic bottle that holds a sample of interstellar material. However, because the bottle has a leak-the ions slowly lose energy to the thin upper atmospherethe material in the bottle is a balance between this leak and the rate at which it is being filled. At present, the intensity of ACR oxygen inside the bottle is about 400 times greater than in interplanetary space. The rate of flow into the bottle varies with the interplanetary ACR intensity, and as a result, the intensity of ions trapped in the bottle varies by perhaps a factor of $\sim 1000$ over the solar cycle.

SAMPEX observations over the next few years should provide an opportunity to use this third radiation belt to study magnetospheric processes and to examine the elemental and isotopic composition of a sample of interstellar matter that, on a Galactic scale, is located right in our own back yard.

\section{References}

Adams, J. H., Jr., et al., The charge state of the anomalous component of cosmic rays, Astrophys. J. Lett., 375, L45, 1991.

Blake, J. B., and L. M. Friesen, A technique to de termine the charge state of the anomalous lowenergy cosmic rays, Proc. 15th Int. Cosmic Ray Conf. (Plovdiv), 2, 341, 1977.

Cummings, A. C., and E. C. Stone, Composition, gradients, and temporal variations of the anomalous cosmic ray component, in Proceedings of the 6 th International Solar Wind Conference, edited by V. J. Pizzo, T. E. Holzer, and D. G. Sime, NCAR Tech. Note NCAR/TN-306, 2, 599, 1987.

Cummings, A. C., E. C. Stone, and W. R. Webber, Estimate of the distance to the solar wind termination shock from gradients of anomalous cosmic ray oxygen, J. Geophys. Res., 98, 15,165, 1993a. Cummings, J. R., A. C. Cummings, R. A. Mewaldt, R. S. Selesnick, E. C. Stone, and T. T. von Rosenvinge, New evidence for geomagnetically trapped anomalous cosmic rays, Geophys. Res. Lett., 20, 2003, 1993b.

Fisk, L. A., B. Kozlovsky, and R. Ramaty, An interpretation of the observed oxygen and nitrogen enhancements in low-energy cosmic rays, Astrophys. J. Lett., 190, L35, 1974.

Gloeckler, G., J. Geiss, H. Balsiger, L. A. Fisk, A. B. Galvin, F. M. Ipavich, K. W. Ogilvie, R. von Steiger, and B. Wilken, Detection of interstellar pick-up hydrogen in the solar system, Science, 261, 70, 1993.

Grigorov, N. L., M. A. Kondratyeva, M. I. Panasyuk, Ch. A. D. Tretyakova, J. H. Adams, Jr., J. B. Blake, M. Shulze, R. A. Mewaldt, and A. J. Tylka, Evidence for trapped anomalous cosmic ray oxygen ions in the inner magnetosphere, Geophys. Res. Lett., I8, 1959, 1991.

Gurnett, D. A., W. S. Kurth, S. C. Allendorf, and R. L. Poynter, Radio emission from the heliopause triggered by an interplanetary shock, Science, $262,199,1993$.

Pesses, M. E., J. R. Jokipii, and D. Eichler, Cosmic ray drift, shock wave acceleration, and the anomalous component of cosmic rays, Astrophys. J. Lett., 246, L85, 1981. 